\title{
Change of perspectives (News Performance)
}

\section{AUTHOR}

Edda Humprecht

\section{KEYWORDS}

news coverage, journalism, viewpoint diversity, plurality, deliberation

FIELD OF APPLICATION/THEORETICAL FOUNDATION Analyses of change of perspectives are theoretically linked to the news performance and democratic functions of the media (McQuail, 1992). This construct is related to viewpoint diversity and the normative expectation that different views should be presented in news coverage (Napoli \& Gillis, 2008). In addition, more recent analysis focus on different perspective articulated in user comments, often linked to theories of deliberation (Baden \& Springer, 2015).

\section{REFERENCES/COMBINATION WITH OTHER METHODS OF DATA COLLECTION}

Perspective change in news coverage is measured i) directly (e.g., by asking whether change of perspective is presented in an article) or i) indirectly by coding different perspective (e.g. statements including different viewpoints). Indirect measures can also be used in automated approaches (Möller et al., 2018).

\section{EXAMPLE STUDIES:}

Baden \& Springer (2014); Humprecht (2016)

\section{REFERENCES}

Baden, C., \& Springer, N. (2014). Com(ple) menting the news on the financial crisis: The contribution of news users' commentary to the diversity of viewpoints in the public debate. European Journal of Communication. https://doi.org/10.1177/0267323114538724
Baden, C., \& Springer, N. (2015). Conceptualizing viewpoint diversity in news discourse. Journalism, 1-19. https:// doi.org/10.1177/1464884915605028

Humprecht, E. (2016). Shaping Online News Performance. In Palgrave Macmillan. Palgrave Macmillan UK. https:// doi.org/10.1007/978-1-137-56668-3

McQuail, D. (1992). Media Performance: Mass Communication and the Public Interest. Sage Publications.

Möller, J., Trilling, D., Helberger, N., \& van Es, B. (2018). Do not blame it on the algorithm: an empirical assessment of multiple recommender systems and their impact on content diversity. Information Communication and Society, 21(7), 959-977. https:// doi.org/10.1080/1369118X.2018.1444076

Napoli, P., \& Gillis, N. (2008). Media Ownership and the Diversity Index: Outlining a Social Science Research Agenda (No. 5; McGannon Center Working Paper Series). 


\begin{tabular}{|c|c|c|c|c|}
\hline Author(s) & Sample & Unity of Analysis & Values & Reliability \\
\hline $\begin{array}{l}\text { Baden \& } \\
\text { Springer } \\
\text { (2014) }\end{array}$ & $\begin{array}{l}\text { Content type: On- } \\
\text { line news cover- } \\
\text { age on selected } \\
\text { key events and } \\
\text { user comments } \\
\text { Outlet/country: } 5 \\
\text { German newspa- } \\
\text { pers (Süddeut- } \\
\text { sche Zeitung, } \\
\text { Die Welt, TZ, Die } \\
\text { Zeit, Spiegel) } \\
\text { Sampling period: } \\
\text { Feb- July } 2012 \\
\text { Sample size: } 42 \\
\text { news articles, } 384 \\
\text { user comments }\end{array}$ & $\begin{array}{l}\text { News article: max. } \\
2 \text { main interpretati- } \\
\text { ve frames (the text's } \\
\text { 'central organizing } \\
\text { idea') } \\
\text { User comments: } \\
\text { main frame }\end{array}$ & $\begin{array}{l}\text { Object of problem definition } \\
\text { Logic of evaluation: } \\
\text { inspired (Good is what is } \\
\text { true, divine \& amazing) } \\
\text { popular (Good is what the } \\
\text { people want) } \\
\text { moral (Good is what is soci- } \\
\text { al, fair, \& moral) } \\
\text { civic (Good is what is accep- } \\
\text { ted \& conventional) } \\
\text { economic (Good is what is } \\
\text { profitable \& creates value) } \\
\text { functional (good is what } \\
\text { works) } \\
\text { ecological (good is what is } \\
\text { sustainable \& natural) } \\
\text { Logic of (inter)action: belie- } \\
\text { ving (interactions between } \\
\text { the mind \& the world) } \\
\text { desire (interaction btw the } \\
\text { mind \& objects) } \\
\text { ought (interaction btw the } \\
\text { mind \& people) } \\
\text { negotiation (interaction btw } \\
\text { people \& the social world) } \\
\text { exchange (interactions btw } \\
\text { people \& objects) } \\
\text { technology (interactions } \\
\text { btw objects \& the world) } \\
\text { life (interactions btw people } \\
\text { \& the natural world) }\end{array}$ & $\begin{array}{l}\text { Authors co- } \\
\text { ded cover- } \\
\text { age consen- } \\
\text { sually } \\
\text { User com- } \\
\text { ments: } \\
\text { M(Holsti) = } \\
0.78 \\
\text { Problem } \\
\text { definition's } \\
\text { object: Hol- } \\
\text { sti=0.60 } \\
\text { Logic of Ac- } \\
\text { tion: Holsti } \\
=0.56 \\
\text { Evaluation } \\
\text { logic: Hol- } \\
\text { sti=1 }\end{array}$ \\
\hline
\end{tabular}




\begin{tabular}{|c|c|c|c|c|}
\hline Author(s) & Sample & Unity of Analysis & Values & Reliability \\
\hline $\begin{array}{l}\text { Hum- } \\
\text { precht } \\
(2016)\end{array}$ & $\begin{array}{l}\text { Content type: } \\
\text { Political routine- } \\
\text { period news } \\
\text { Outlet/ country: } \\
48 \text { online news } \\
\text { outlets from six } \\
\text { countries (CH, } \\
\text { DE, FR, IT, UK, } \\
\text { US) } \\
\text { Sampling period: } \\
\text { June - July } 2012 \\
\text { Sample size: N= } \\
1660\end{array}$ & $\begin{array}{l}\text { Unit of analysis: } \\
\text { Political news items } \\
\text { (make reference to } \\
\text { a political actor, e.g. } \\
\text { politician, party, } \\
\text { institution in head- } \\
\text { line, sub-headline, } \\
\text { in first paragraph } \\
\text { or in an accompa- } \\
\text { nying visual) } \\
\text { News items are all } \\
\text { journalistic articles } \\
\text { mentioned on the } \\
\text { front page ('first } \\
\text { layer' of the web- } \\
\text { site) that are linked } \\
\text { to the actual story } \\
\text { (on second layer of } \\
\text { website) }\end{array}$ & $\begin{array}{l}\text { Only one perspective (be- } \\
\text { cause underlying topic is } \\
\text { uncontroversial) } \\
\text { One perspective (of a deba- } \\
\text { ted/controversial issue, no } \\
\text { opposition voice) } \\
\text { Different perspectives mentio- } \\
\text { ned (different sides, voices, } \\
\text { camps, perspectives men- } \\
\text { tioned but not elaborated) } \\
\text { Co-presence of speakers with } \\
\text { opposing views (expressed in } \\
\text { separate utterances) in the } \\
\text { same article. Story shows } \\
\text { clear attempt at giving a } \\
\text { balanced, fair account of } \\
\text { debated/controversial issue } \\
\text { by including diverse view- } \\
\text { points and statements) }\end{array}$ & $\begin{array}{l}\text { Cohen's } \\
\text { kappa: } \mathrm{M}= \\
0.64\end{array}$ \\
\hline
\end{tabular}

